\title{
Through the looking glass: How the relationship between an object and its reflection affects the perception of distance and size
}

\author{
Luke A Jones \\ Division of Psychology, School of Psychological Sciences, University of Manchester, Oxford Road, \\ Manchester M13 9PL, UK; e-mail: luke.jones@manchester.ac.uk
}

\section{Marco Bertamini}

School of Psychology, University of Liverpool, Eleanor Rathbone Building, Liverpool L69 7ZA, UK Received 5 April 2006, in revised form 30 April 2007; published online 22 November 2007

\begin{abstract}
This is the first study to test the extent to which reflections help locate objects in space and perceive their size. For planar mirrors, the relative size of a target and its reflection is informative about the absolute distance of the target in units of the distance between target and mirror surface. When the target is near the mirror, target and reflection are similar in size; as the target moves away from the mirror, the difference in size increases. Observers saw a pair of objects in front of a mirror and judged relative size and distance (separately). Other visual cues to size and distance were eliminated, except lateral offset, which was tested in experiment 3. Experiment 2 controlled for the presence of directional feedback. Results showed orderly psychophysical functions for both size and distance with steeper slopes for distance judgments. In experiments 4 and 5 stereograms were used. Even when binocular information was present, the additional cue provided by reflections increased the accuracy of size and distance judgments. The same pattern of results was observed in the absence of feedback.
\end{abstract}

"There are two ways of spreading light: to be the candle or the mirror that reflects it." from "Vesalius in Zante (1564)" by Edith Warton (1902)

\section{Introduction}

Surfaces can reflect light in more than one way. On opaque surfaces most light is diffused, ie it is scattered isotropically in all directions. However, on some surfaces a component of incident light is reflected anisotropically within a limited range of directions. Of particular interest are planar specular surfaces for which a large component of light is reflected and virtual images are formed without magnification or distortions. These surfaces - flat mirrors - have become common in our domestic and built environment.

The virtual world in a mirror provides all the depth cues of the real world, including binocular disparity, and is fundamentally unlike pictorial representations. When we look into a mirror, we see another world, as if we were looking through a portal. Indeed, it is probably this property (in addition to narcissistic indulgence) that has made mirrors so alluring to humans throughout history. But do reflections affect how we perceive the world - that is, not the virtual world but the world being reflected?

\subsection{Previous reflection studies}

There are various literature references dealing with reflections; for instance, there is a debate why mirrors reverse left and right but not top and bottom (eg Gardner 1967; Ittelson 1993; Gregory 1997; a series of empirical studies is in Ittelson et al 1991). Mirrors have also been used to study the development of conscious awareness (eg Gallup 1998; Povinelli 2000). A recent study demonstrated that surface reflections are crucial to our perception of the surface texture and material composition of objects. Different materials 
have varying reflecting properties and give rise to differing surface reflections, from a dull fuzzy highlight to a glossy mirror-like surface (Fleming et al 2003). This finding suggests sophisticated processing within the visual system as the appearance of surface reflections is confounded by the effects of illumination.

How people reason about mirror reflections has been investigated within the domain of naive optics. People exhibit systematic errors in their reasoning. They tend to overestimate what is visible in a mirror (Croucher et al 2002; Bertamini et al 2003b; Hecht et al 2005) - an error that is dependent on the spatial relationship between the observer and the mirror (Jones and Bertamini 2007). People also fail to properly take into account the location of the viewpoint. For example, when observers describe scenes in which both an actor and a mirror are present, observers tend to believe that what is visible to them in the mirror is also visible to the actor, a phenomenon that has been termed the 'Venus effect' (Bertamini et al 2003a).

Bertamini and Parks (2005) analysed what happens to the size of images on mirrors and compared this with people's beliefs regarding these images. This type of explicit knowledge is remarkably poor. For instance, people tend to believe that the image we see of our own self on the surface of a mirror gets smaller as our distance from the mirror increases. It actually stays the same. Conversely, people tend to believe that the image of a stationary target on a stationary mirror will remain the same when the viewpoint changes. It does not, because target and viewpoint are not coupled. This suggests that people focus on the target location and fail to appreciate the role of the viewpoint (consistent with the Venus effect).

In a recent study, Higashiyama and Shimono (2004) examined spatial judgments about virtual objects. Participants were required to judge the perceived size and distance of virtual objects of a series of targets placed at a range of distances. Participants viewed real objects that were placed behind them by looking at a handheld mirror in front of them. Both planar and convex mirrors were used. Size constancy and orderly distance judgments were obtained up to a distance of $45 \mathrm{~m}$. The authors found that the ratio of perceived size to perceived distance was described as a power function of virtual angle. Results were similar for both planar and convex mirrors, although there was a tendency for virtual images in convex mirrors to be judged as farther away. Essentially, it was found that people were able to make size and distance judgments comparable in accuracy to the judgment of real objects even when the mirror had a strong convex curvature.

Although important in relation to people's everyday reasoning about mirrors, the papers described above were not concerned directly with the sensitivity of observers to the spatial information provided by reflections about the objects reflected. In the naive-optics studies, reflections were often not presented as part of experiments or were not manipulated systematically (Bertamini et al 2003b; Bertamini and Parks 2005; Hecht et al 2005). Reflections were presented in Higashiyama and Shimono's study (2004); however, participants were ultimately required to make judgments about virtual objects and did not directly view their real counterparts. What remains to be addressed, then, is whether information derived from reflective surfaces influences our perception of the world. People make accurate judgments regarding the size and distance of virtual images of objects on a natural terrain, which is a testament to the similarity between real and virtual objects. Moreover, the real and virtual couplet is linked in other ways, so it is reasonable to suppose that the relationship ought to be informative about the layout of the scene being reflected.

In a series of experiments, we examined the ability of observers to make judgments about objects viewed together with their reflections. By removing or manipulating the presence of other depth cues we were able to systematically evaluate the contribution of reflections to the perception of size and distance within scenes. 


\subsection{Reflections and shadows}

The potential role of reflections in our perception of the real world has been hitherto overlooked. This is unsurprising as other sources of information about distance and size, such as binocular parallax, motion parallax, occlusion, relative height and size, texture gradient, linear perspective, and atmospheric perspective, are more common and more easily demonstrated. However, there has been recent interest in another source of information that has also been overlooked in the past: shadows. In the case of shadows, systematic investigations have revealed that they can affect perception of shape and motion (eg Knill 1997; Mamassian et al 1998; Elder et al 2004).

Just as cast shadows are closely linked by optical principles to the objects that cast them, reflections in mirrors are linked to the objects that are reflected. Both cast shadows and reflections can be spatially detached from the originating objects. In the case of undistorted reflections from planar mirrors, reliable information regarding object shape is present in the reflection. Conversely, in the instance of distorted reflections of visible objects, there are potentially salient cues regarding the properties of the reflecting surface. Therefore, reflections share many characteristics with cast shadows and provide similar, if not richer, information regarding spatial configuration, shape, and distance.

The investigation of cast shadows is useful for our investigation of reflections. Similar research questions can be asked, although important differences exist. The properties of shadows are dependent on the illuminant as well as the object and the surface onto which they are cast. For instance, multiple illuminants can give rise to multiple shadows, and the properties of the illuminant determine whether shadow edges are hard or soft. Compared with reflections, there is considerable noise in the information provided by shadows. Although reflections require an appropriately placed specular surface, for planar surfaces the virtual object matches the original object in shape under an isometric transformation. ${ }^{(1)}$ That is, metric information is preserved in the virtual object. With respect to the relationship between objects and their reflections, the illuminant has no role to play: multiple illuminants do not generate multiple virtual objects.

The illuminant is also responsible for another important difference between shadows and reflections. The ratio of the size of the object to the size of its reflection decreases as the object gets closer to the mirror. That is, the size difference between an object and its reflection decreases as the object approaches the mirror. Similar information is in theory available from shadows. If we imagine the mirror replaced by an opaque surface, such as a pale coloured wall, then under certain conditions the ratio of the size of the object to the size of its shadow would also diminish as the object approached the wall. However, for this to apply, the light source would have to be close to the object. If the light source were near infinity (such as the sun), then the difference in the size of the shadow cast by an object at different distances from the wall/light source would be infinitesimally small. Moreover, the light source would have to be located at (or extremely close to) the viewpoint (a form of extramission!). If the light source is offset from the viewpoint, the relationship of object size and shadow size becomes exceedingly complex. By contrast, neither of these factors (distance of light source or offset of light source) are of importance when considering the potential information provided by reflections.

(1) By definition a virtual image is generated by a planar mirror behind the mirror surface. Although rays of light seem to come from behind the mirror, light spreads and exists only in front of the mirror. Therefore the virtual object does not exist as a physical object, but it exists with full rights as a visual object. The match between real and virtual objects preserves metric information but the two are not identical, they are related by a mirror reflection (they are enantiomorphs) like, for instance, the left and right hands. 
Despite the noise in the information that shadows provide compared to reflections, shadows have an important advantage. This advantage is based not on geometry but on ecology. Shadows are more common in our environment by far, especially from an evolutionary perspective. The possibility of multiple illuminants is a recent factor, and indeed the visual system assumes light sources are singular and located above (eg Kleffner and Ramachandran 1992). Speculation suggests that reflections were comparatively rare in our evolutionary past, even when we consider water as a specular surface. It is rare for a naturally occurring water surface to provide clear reflections. Water sources that are fast moving (such as rivers) will have surface reflections that are disturbed. Slow, stagnant, or muddy water tends to be murky. Additionally, a water surface, because of gravity, provides reflections only of objects located above it, usually the sky, or objects located directly next to the edge of the water and leaning over it. A full consideration of the evolutionary role in the use of reflections is beyond the scope of this article. One way in which a future experimental series could explore this issue would be to run a similar methodology as described here but with imperfect reflective surfaces rather than planar mirrors.

In summary, given the limited presence of planar mirrors in our evolution, one might assume that little about mirrors has been internalised by the visual system. However, before we can address the innate versus learned aspects of the problem, the degree to which the observers can exploit information from reflections must be tested empirically. This is the focus of our current study.

\subsection{Reflections as information about space and position}

In general terms, when an object is close to a mirror surface the object and its reflection will appear similar in size. The further an object is from the mirror, the larger the difference between them. This fact is related to foreshortening: the front and back of a cube look similar if their distance is small relative to the viewpoint distance, but the difference gets larger if the observer moves nearer (or the two faces of the cube move apart) (eg Nicholls and Kennedy 1993; Young and Kubovy 1999). ${ }^{(2)}$

We discuss the information about size and distance available from reflections with the help of figure 1 (see also Bertamini and Parks 2005). It is useful to think of the real object (target) and the virtual object as a pair of visible objects. That the virtual object inhabits a virtual world is irrelevant with respect to visual information. The important point is that a real target and a virtual target inside a mirror are objects of exactly the same physical size. Therefore the ratio between the heights of their two images $\left(r=\right.$ image/image $\left.{ }^{\prime}\right)$ is inversely related to the ratio between their two distances from the same vantage point $\left(r=d^{\prime} / d\right)$. We define $k$ as the distance between the object and the surface of the mirror. The distance of the virtual object is the distance of the real object plus twice $k$. This is because the virtual target is always at the same distance inside the mirror as the real target is from the mirror. From this we derive the relationship between $d$ and $k$ :

$$
r=\frac{2 k}{d}+1 ; \quad d=\frac{2 k}{r-1} .
$$

When the mirror is not aligned with the target we need to know the angle $\theta$. The diagram in figure 1 shows that $d$ can be decomposed into $d_{1}$ and $d_{2}$, and $d^{\prime}=c+k$ is the distance to the virtual target inside the mirror. Note that $d^{\prime}=d+2 k$ is not true any longer. Since $b=k \sin \theta$ and $d_{2}=k \cos \theta$ we can express

(2) For instance, superman appears to pop out of a comic page when he is drawn flying towards the reader, partly because his hands are drawn large and his feet small. For both superman and for the cube the effect of foreshortening relies on familiarity with the shape of the object. 


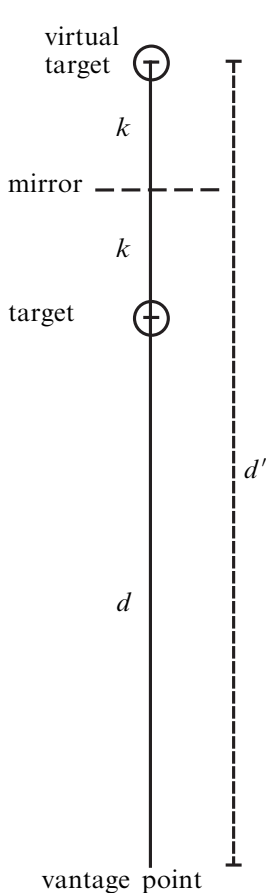

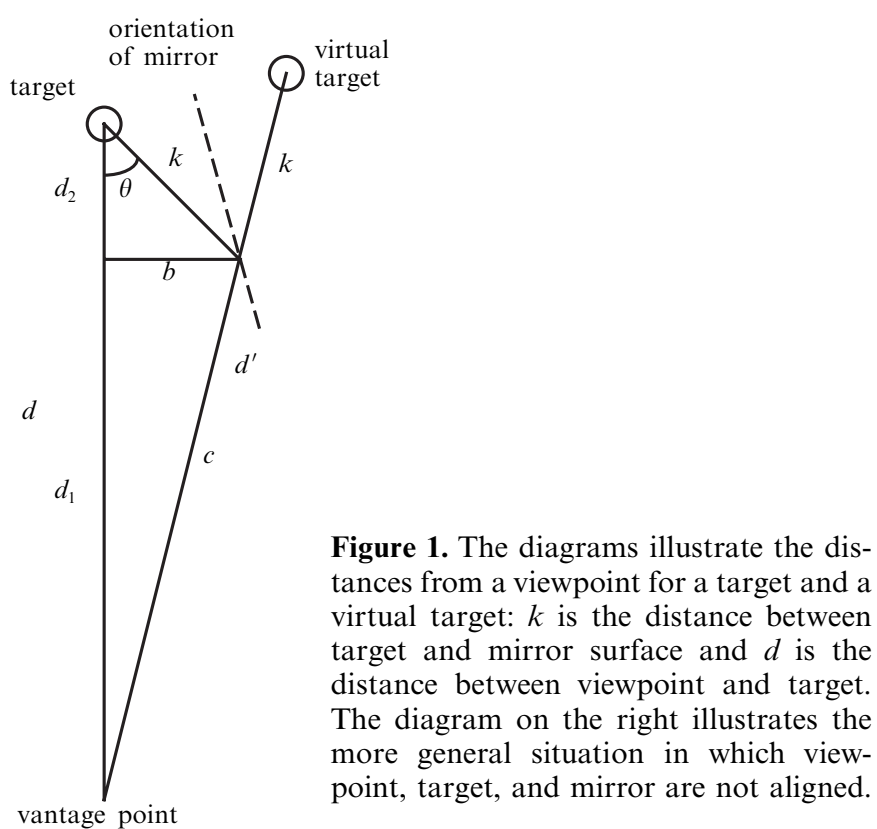

$c$ in terms of $d$ and $k$ :

$$
\begin{array}{ll}
c^{2}=k^{2} \sin \theta^{2}+(d-k \cos \theta)^{2} ; & c^{2}=k^{2}+d^{2}-2 d k \cos \theta ; \\
c=\left(k^{2}+d^{2}-2 d k \cos \theta\right)^{1 / 2} ; & d^{\prime}=k+\left(k^{2}+d^{2}-2 d k \cos \theta\right)^{1 / 2} .
\end{array}
$$

Let us express $r$ (as before) as the ratio between the distances from the vantage point to the virtual and the real target:

$$
\begin{aligned}
& r=\frac{k+\left(k^{2}+d^{2}-2 d k \cos \theta\right)^{1 / 2}}{d}, \quad(r d-k)^{2}=k^{2}+d^{2}-2 d k \cos \theta, \\
& r^{2} d^{2}+k^{2}-2 r d k-k^{2}-d^{2}+2 d k \cos \theta=0, \\
& \left(r^{2}-1\right) d^{2}+2 d k(\cos \theta-r)=0, \quad d^{2}=\frac{-2 d k(\cos \theta-r)}{r^{2}-1}, \quad d=2 k \frac{r-\cos \theta}{r^{2}-1} .
\end{aligned}
$$

This formula is more general, and when $\theta=180^{\circ}$ the simpler version can be derived

$$
d=\frac{2 k(r+1)}{(r-1)(r+1)}=\frac{2 k}{r-1} \text {. }
$$

In summary, when both a target and its reflection are available, their relative size is informative about the absolute distance of the target in units of the distance $k$ between target and specular surface. The basic relationship is given by $d=2 k /(r-1)$, where $d$ is the distance of the viewpoint from the target object, and $r$ is the ratio of the apparent sizes of target and virtual target.

This relationship is of limited importance with respect to absolute distance and absolute size because it still requires knowledge of the unknown distance $k$. Relative distance and relative size, on the other hand, are fundamentally different. Relative information is available even without knowing $k$. Consider the case of two objects (A and B) 
at unknown distances $\left(d_{\mathrm{A}}\right.$ and $\left.d_{\mathrm{B}}\right)$ in front of a mirror in the frontoparallel plane:

$$
d_{\mathrm{A}}+k_{\mathrm{A}}=d_{\mathrm{B}}+k_{\mathrm{B}}, \quad d_{\mathrm{A}}-d_{\mathrm{B}}=k_{\mathrm{B}}-k_{\mathrm{A}} .
$$

From what we have seen before, we can write $k$ as a function of $r$ and $d$ (for both object $\mathrm{A}$ and object $\mathrm{B})$ :

$$
k_{\mathrm{A}}=\frac{1}{2}\left(r_{\mathrm{A}}-1\right) d_{\mathrm{A}} ; \quad k_{\mathrm{B}}-\frac{1}{2}\left(r_{\mathrm{B}}-1\right) d_{\mathrm{B}} .
$$

We can then have a formula in which $k$ does not appear

$$
\begin{aligned}
& d_{\mathrm{A}}-d_{\mathrm{B}}=\frac{1}{2}\left(r_{\mathrm{B}}-1\right) d_{\mathrm{B}}=\frac{1}{2}\left(r_{\mathrm{A}}-1\right) d_{\mathrm{A}} \\
& d_{\mathrm{A}}=\frac{r_{\mathrm{B}}+1}{2} \frac{2}{r_{\mathrm{A}}+1} d_{\mathrm{B}}=\frac{r_{\mathrm{B}}+1}{r_{\mathrm{A}}+1} d_{\mathrm{B}} .
\end{aligned}
$$

This means that, if two values of $r$ are available for two objects, then the ratio of their physical heights and the ratio of their distances from the same vantage point are theoretically available without the need to estimate either $k_{\mathrm{A}}$ or $k_{\mathrm{B}}$. The question is whether people make use of this information.

In our experiments two targets were present in front of the same mirror, and both their reflections were visible. Relative information means that the units of measurement are irrelevant and the value of $k$ does not matter. For an unknown value of $k$, it is still possible to know if target $\mathrm{A}$ is, say, $10 \%$ farther away than target $\mathrm{B}$ from the same vantage point. Our study therefore concentrated on relative size and distance judgments.

It should be emphasised that the logical link between size and distance does not imply that the two are linked in perception (Gruber 1954; Epstein et al 1961). From an ecological perspective, some information about size is present in images independently of distance (eg the horizon ratio-Sedgwick 1983; Bertamini et al 1998). It has also been found that despite tremendous compression in perceived pictorial distance, perceived size was not affected by this distortion (Rogers 2003). Because of this, we employ both perceived distance and perceived size as dependent variables in our studies.

\section{General method and programme of experiments}

We created 3-D scenes using the graphic package MAXON Cinema 4D (Maxon, $\mathrm{GmbH}$, Friedrichsdorf, Germany). Figure 2 shows two semitransparent spheres in front of a planar mirror. We used spheres because they are objects of unfamiliar size, and we made them semitransparent to maximise visual information, in the sense that the frame and the reflected images are visible even when partly occluded by the objects in front.

In the set of experimental stimuli, one object was kept unchanged and acts as the standard, the second object changed in both size and distance. The standard was colour-coded as blue and the comparison as yellow. In the absence of a reflection it would be impossible to know whether the difference in apparent height (visual angle) between the two targets is due to a difference in size or a difference in distance. ${ }^{(3)}$ However, on the basis of the relationship between each target and its corresponding reflection, the ambiguity is eliminated. In figure 2 the sphere on the right is $10 \%$ nearer and $20 \%$ shorter than the sphere on the left. To be more precise, the analysis of this figure is complicated by the horizontal offset of both targets (the angle $\theta$ in figure 1), but this factor becomes weaker for small offsets and viewing distances that are large compared to $k$. For example, taking the scene shown in figure 2, the calculation given in the figure caption shows that this factor $(\theta)$ has an effect too small to be relevant

(3) Note that the objects are floating at eye level so that the horizon ratio cannot be used to gather information about size (although the horizon ratio does specify something about the size of the mirror if it is perceived as standing on the ground) (Sedgwick 1983; Bertamini et al 1998). 


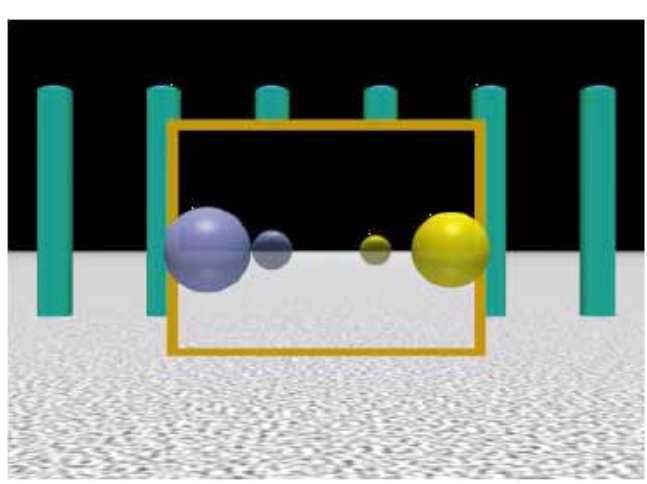

(a)

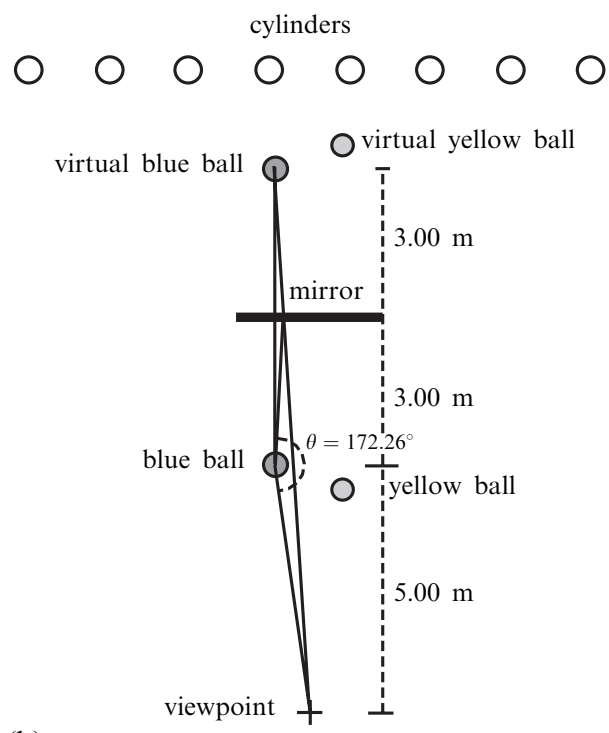

(b)

Figure 2. (a) Example of stimuli for experiment 1. A blue and a yellow semitransparent sphere are floating at eye-height in front of a mirror. The mirror is in the frontoparallel plane, and it shows the reflections of the spheres. The terrain has a noisy texture that provides slant information but no familiar size. The relative distance from the observer and therefore also the relative height of the two objects is specified by the relationship between the image of the target and the image of the virtual target. (b) Plan view of scene layout. Let us evaluate the role of $\theta$ which is $180-\theta_{1}$. We start with the equation for when the viewpoint and objects are not exactly aligned: $d=2 k(r-\cos \theta) /\left(r^{2}-1\right)$. To calculate $\theta$ we must first calculate the offset of the target object from the viewpoint $\left(\theta_{1}\right)$, we calculate this from a triangle plotted between the actual viewpoint, the target object and the aligned viewpoint, thus, $\cos \theta_{1}=5 / x$. To do this we need $x$ which is the distance between the actual viewpoint and the target object, $x^{2}=5^{2}+0.68^{2}, x=5.046$. Therefore $\cos \theta_{1}=5 / 5.046$, then $\theta_{1}=\cos ^{-1} 0.99$ and $\theta_{1}=7.74^{\circ}$; we can now calculate $\theta$ : $\theta=180^{\circ}-7.74^{\circ}=172.26^{\circ}$. We now find $d=2 k(r-\cos \theta) /\left(r^{2}-1\right), d=2 k(r-0.99) /\left(r^{2}-1\right)$ which is approximately equal to $d=2 k /(r-1)$. Therefore the fact that the viewpoint and object are not aligned has a negligible effect (relative to the likely noise). Note that, although the distances are given in metres, this is a simulated scene so the scale is actually arbitrary. A colour version of this and figures 5 and 7 can be found online at http://www.perceptionweb.com/abstract.cgi?id=p5605/.

given the expected noise in the data. Therefore, for simplicity we will use the simpler formula in discussing and analysing our findings.

Whatever the simulated size and distance of the objects, they were positioned so as to occupy the same position in the image. By that we mean that they overlapped the outside frame of the mirror by the same amount (by $2 \mathrm{~mm}$ on the test stimuli). This ensured that the horizontal position of the target and comparison object was unrelated to size and distance. This is not true for the reflections. We looked at the possible role of this factor in experiment 2.

The fact that the standard and the comparison spheres are colour-coded (blue and yellow, respectively) has an additional advantage: the colours make the correspondence between targets and virtual targets unambiguous.

Using stimuli like those in figure 2 we tested the perception of relative distance and relative size of the comparison object. Observers judged to what extent (as a percentage) sphere B was taller or shorter than sphere A. In another condition observers were asked to what extent (as a percentage) sphere B was nearer or farther away from the vantage point than sphere A. In both cases we used a response scale from $-40 \%$ to $+40 \%$, and observers saw a series of images during a session that lasted about $30 \mathrm{~min}$. 
In experiment 1 we tested observers' perceptions of relative size and distances. In addition, we examined the effect of binocular versus monocular viewing of the test stimuli. We investigated this factor because monocular viewing of a 2-D image can lead to a greater sense of depth. The likely reason is the elimination of binocular cues to the flat nature of the image (eg Ames 1925; Schlosberg 1941). When viewing the computer screen binocularly, the eyes converge on the flat surface of the computer monitor, thus providing depth cues that are in conflict with the monocular depth cues. Therefore, it is conceivable that monocular viewing will increase the perceived depth of the scene and make the mirror in the scene appear more realistic, increasing the likelihood that the depth information in the mirror will be used as such. ${ }^{(4)}$ In experiment 2, we looked at the role of horizontal offset between the spheres and their reflections, and in experiment 3 we assessed any additional benefit of the reflections when binocular disparity information was available for the visual scene. To this end we turned our stimuli into stereograms. In experiment 4 we explored the results of experiment 3 further by presenting only monocular information inside the mirror whilst binocular disparity was present for the target objects and the rest of the visual scene.

\section{Experiment 1: Relative judgments of size and distance}

In experiment 1 observers judged the relative size and distance of two spheres floating in front of a planar mirror. The spatial layout of the 3-D scene is illustrated in figure 2. We were interested in whether and to what extent people could judge size and distance from the presence of reflections in a planar mirror.

We compared monocular and binocular viewing conditions. It is well-known that depth cues that are present in 2-D pictorial representations (such as a photograph or a computer screen) are more powerful and salient when viewed monocularly, because the conflict from binocular information about the surface of the medium (the computer screen) is eliminated (eg Ames 1925; Schlosberg 1941). Perhaps monocular viewing would lead to better usage of the depth cues in the computer image, because this is fundamentally a monocular cue. That is, the relative size of the object compared to its reflection is specified monocularly. To reduce the influence of the surface of the computer screen in both monocular and binocular viewing conditions we also used a screen covering the monitor, with an aperture $14.3 \mathrm{~cm}$ in diameter.

\subsection{Method}

3.1.1 Participants. Sixteen Liverpool University undergraduates participated to attain course credits. They had normal or corrected-to-normal vision.

3.1.2 Apparatus and procedure. We created two series of 3-D scenes. Two spheres, one blue and one yellow, were located in front of a planar mirror. The blue sphere (standard) was always the same size and distance from the mirror (figure 2). In one series of images the yellow test sphere had a retinal size $10 \%$ smaller than the blue sphere, in the other series is was $10 \%$ larger. In each series, a range of relative sizes and distances of the yellow sphere to blue sphere were used, from $40 \%$ smaller to $40 \%$ larger in increments of $10 \%$. The standard sphere appeared on the left or the right of the yellow sphere with equal probability. The same images were used for both distance and size judgments. All images were $24 \mathrm{~cm}$ wide and $18 \mathrm{~cm}$ high. The experiment was

(4) It should also be mentioned that our test images lack one important feature which would be present in real reflections: the observer should be present in the mirror. We repeated experiment 1 in our lab with a generic human figure located at the viewpoint, so that observers were able to imagine that what they saw was an image of themselves in the mirror (more precisely the image of the individual who took the original photograph, as our stimuli are best conceived as computer simulated photographs). We found that having the observer present in the image did not significantly improve the accuracy of size and distance judgments; if anything it provided a distraction. For the sake of expediency we do not include the data in this report. 
blocked into a size judgment condition and a distance judgment condition. Participants completed both conditions with the order counterbalanced. There were two sessions for each of the size and distance conditions: a monocular viewing condition and a binocular viewing condition, again with the order counterbalanced within each session. In the monocular condition the participants wore an eye patch over their nondominant eye.

All experiments were run on a Macintosh computer in a quiet and darkened room. The programme that displayed the images was developed in $\mathrm{C}$ by the authors. The monitor was a Mitsubishi Diamond90e with a resolution of $1280 \times 1024$ pixels at $75 \mathrm{~Hz}$. A reduction screen was used to obscure the area of the screen surrounding the test images.

Participants made their responses on the computer keyboard by pressing one of nine keys labelled $-40 \%,-30 \%,-20 \%,-10 \%$, same, $+10 \%,+20 \%,+30 \%,+40 \%$. The procedure was self-paced; the participants were free to observe the image for as long as they required to make a decision. Once a response was given, a new test image appeared. To avoid misunderstanding, the computer beeped if the response was in the incorrect direction, ie if the participant gave one of the nearer responses when one of the farther responses was correct (in the distance task), or gave one of the bigger responses when one of the smaller responses was correct (in the size task), no feedback was given on 'same' trials. There were 32 test images in each condition, each one repeated 2 times - a total of 64 trials.

\subsection{Results}

3.2.1 Size judgments. Figure 3a shows mean perceived size difference plotted against actual size difference. Inspection of the data shows that perceived size difference increased orderly and linearly with actual size difference. There also appears to be little effect of viewing condition (monocular versus binocular) on size judgments.
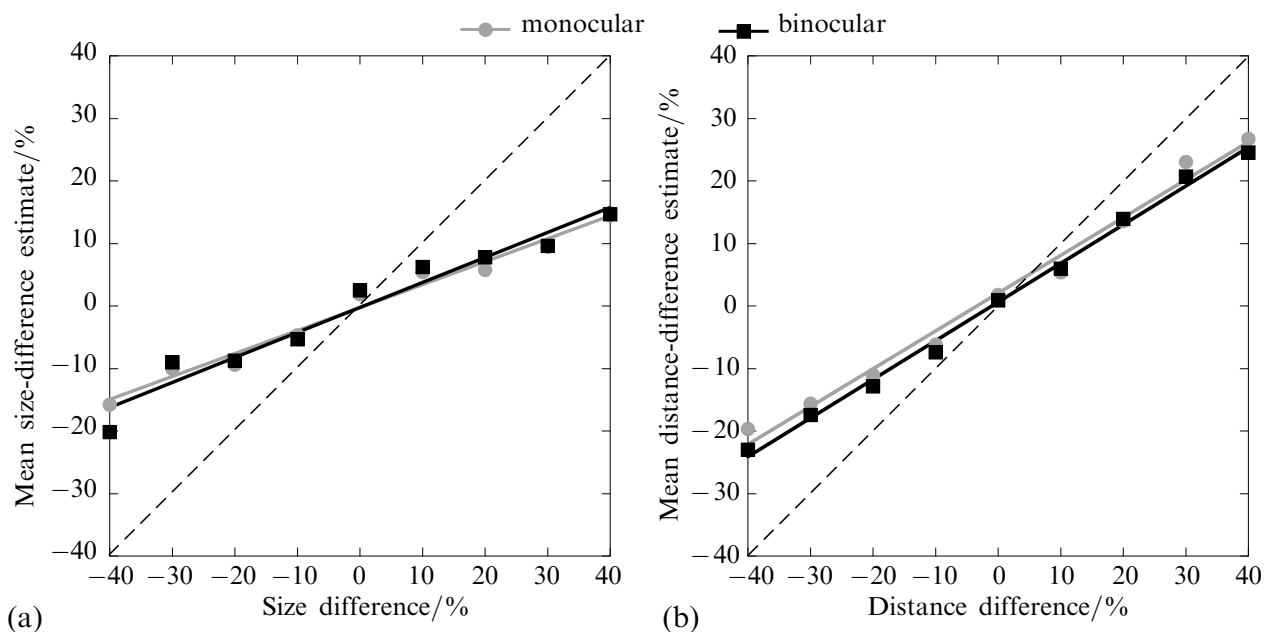

Figure 3. Plot of mean difference estimate against actual difference from experiment 1 . The two panels show size judgments and distance judgments. Data are fitted by linear regression. Slope values: monocular size 0.37 ; binocular size 0.39 ; monocular distance 0.60 ; binocular distance 0.62 .

These suggestions were confirmed by a repeated-measures analysis of variance (ANOVA) with viewing (monocular or binocular), and relative size $(-40 \%$ to $+40 \%$, in $10 \%$ steps) as factors. Relative size was analysed as a linear contrast, as we were interested in the slope of the function. There was a significant effect of relative size $\left(F_{1,15}=31.00, p<0.001\right)$ indicating that participants were sensitive to this information. There was no significant effect of viewing $\left(F_{1,15}=0.005, p=0.940\right)$. There was also no significant viewing $\times$ relative-size interaction $\left(F_{1,15}=0.41, p=0.530\right)$, which 
indicated that the slopes for the monocular and binocular conditions did not differ significantly. The values of the slopes are given in the caption of figure 3 .

Separate analyses for the two subsets of the stimuli with different retinal size led to the same conclusions. Viewing was not significant (in both cases $F_{1,15}<1.0$ ), but relative size was significant $\left(F_{1,15}=21.89, p<0.001\right.$; and $\left.F_{1,15}=22.81, p<0.001\right)$.

3.2.2 Distance judgments. Figure $3 \mathrm{~b}$ shows mean perceived distance difference plotted against actual distance difference. Inspection of the data shows that perceived distance difference increased orderly and linearly with actual distance difference. There also appears to be little effect of viewing condition on distance judgments.

These suggestions were confirmed by a repeated-measures ANOVA with viewing (monocular or binocular) and relative distance $(-40 \%$ to $+40 \%$, in $10 \%$ steps) as factors. As for the size analysis, the factor relative distance was treated as a linear contrast. There was a significant effect of relative distance $\left(F_{1,15}=171.60, p<0.001\right)$. There was also a significant main effect of viewing condition $\left(F_{1,15}=6.34, p<0.05\right)$, but no significant viewing $\times$ relative-distance interaction $\left(F_{1,15}=0.11, p=0.742\right)$. This indicates that the slopes of the monocular and binocular conditions did not differ significantly from each other.

Separate analyses for the two subsets of the stimuli with different retinal size led to the same conclusions. Viewing was not significant (in both cases $F_{1,15}<3.0$ ), but relative size was significant $\left(F_{1,15}=171.70, p<0.001\right.$; and $\left.F_{1,15}=84.53, p<0.001\right)$.

3.2.3 Distance versus size judgments. Figure 3 suggests that the slope for the distance judgments is steeper than that for the size judgments. This suggestion was supported by a repeated-measures ANOVA with the following factors: task (size or distance), viewing (monocular or binocular) and relative-distance/size $(-40 \%$ to $+40 \%$, in $10 \%$ steps). As for the previous analyses, the relative difference was treated as a linear contrast. There was no main effect of task $\left(F_{1,15}=1.95, p=0.18\right)$ but there was a significant interaction between task and relative-distance/size $\left(F_{1,15}=9.57, p<0.01\right)$, indicating that the slopes for the distance and size judgments were significantly different. There were no other significant main effects or interactions with the exception of a main effect of relative-size/distance $\left(F_{1,15}=117.43, p<0.001\right)$, which simply confirmed that participants were sensitive to this information.

\subsection{Discussion}

The results of experiment 1 suggest that people were able to use the information provided by mirror images to make judgments of the size and distance of the objects being reflected. Although performance was far from perfect (see figure 3), participants performed better than chance.

It is also clear from figure 3 that there was limited use of the farthest categories, which is a consistent feature of psychophysical tasks. Looking at the data, it seems that the scale provided sufficient choice to the participants and the range did not constrain performance.

An important additional finding was that distance judgments were more accurate than size judgments (ie the slope was steeper). On first appraisal, this would seem a surprising result because, from the geometry, these two judgments should be linked. Moreover, the same stimuli and the same response scale were used in the two tasks. It is possible that people simply are better at judging distance, but it is also possible that they rely on different cues for different judgments. Although we tried to remove all depth cues in our images, one possible cue to distance was still present.

The horizontal offset of the target object to its reflection varies linearly with depth, such that, as the target approaches the mirror, the horizontal offset is reduced, ie the target gets closer to its reflected image until it eventually occludes it. This cue in itself 
is unlikely to support an accurate quantitative depth judgment, but may have a small but significant effect. Although there is sufficient information from the relationship between targets and reflections, it is possible that this additional horizontal offset is responsible for the superior performance for distance judgments over size judgments. Experiment 3 was designed to test directly this additional cue of horizontal offset.

\section{Experiment 2: Relative judgments of size and distance in the absence of feedback}

In experiment 1 the participants were given directional feedback during testing. This was done to avoid the possibility that participants would misunderstand the meaning of the positive and negative signs in their responses. We were particularly worried about people thinking in terms of distance to the mirror rather than distance from the observer. Having now found an above-chance performance in experiment 1 we thought it prudent to conduct a control experiment for the presence of feedback. We therefore repeated the monocular viewing condition of experiment 1 without the presence of directional feedback.

\subsection{Method}

4.1.1 Participants. Ten Liverpool University undergraduates (who did not participate in any of the previous experiments) participated to attain course credits. They had normal or corrected-to-normal vision.

4.1.2 Apparatus and procedure. These were identical to those in experiment 1, but with the absence of any performance feedback. Participants viewed all images monocularly.

\subsection{Results}

4.2.1 Size and distance judgments. Figure 4 shows mean perceived size difference plotted against actual size difference (a), and mean perceived distance difference plotted against actual distance difference (b). Inspection of the data shows that judgment of size difference increased orderly and linearly with actual size difference, and that judgment of distance difference increased orderly and linearly with actual distance difference. Additionally, figure 4 suggests that the slope for the distance judgments is steeper than that for the size judgments.

All these suggestions were confirmed by a repeated-measures ANOVA with task (size of distance), and relative-size/distance $(-40 \%$ to $+40 \%$, in $10 \%$ steps) as factors. As in the previous analyses the relative difference was treated as a linear contrast.
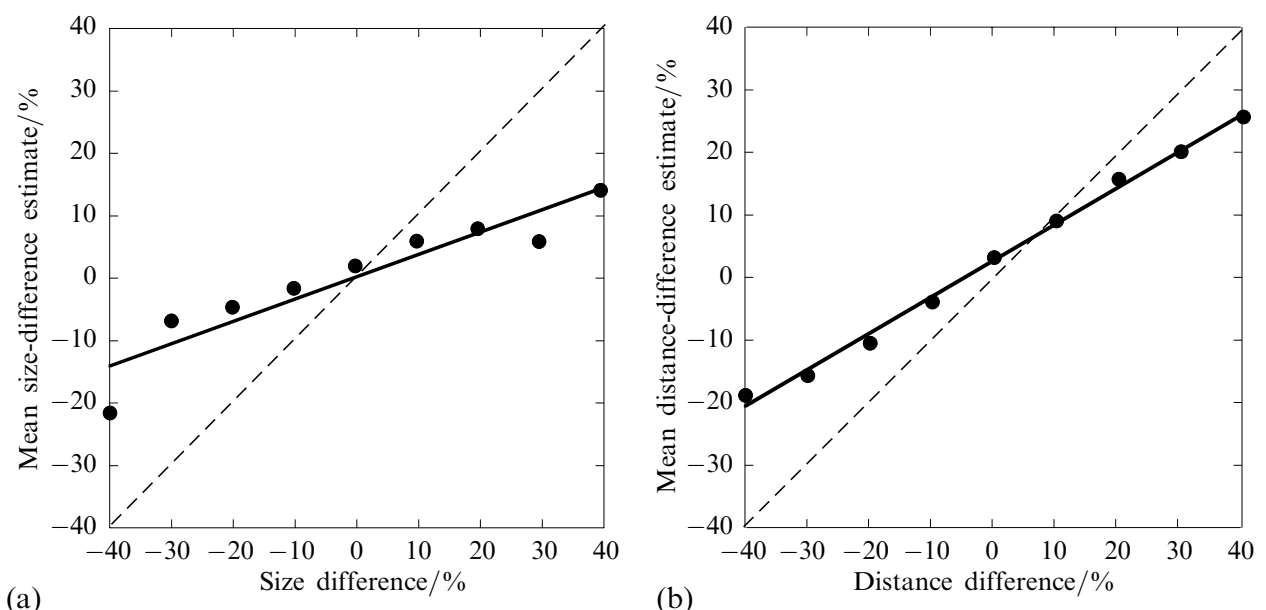

Figure 4. Plot of mean difference estimate against actual difference from experiment 2. The two panels show size judgments and distance judgments. Data are fitted by linear regression. Slope values: size judgments 0.37 ; distance judgments 0.59 . 
There was no main effect of task $\left(F_{1,9}=3.52, p=0.09\right)$, but there was a significant interaction between task and relative-distance/size $\left(F_{1,9}=8.19, p<0.05\right)$, indicating that the slopes for the distance and size judgments were significantly different. There was also a main effect of relative-size/distance $\left(F_{1,9}=75.20, p<0.001\right)$, which confirmed that participants were sensitive to this information.

\subsection{Discussion}

The results of experiment 2 show an identical pattern to those of experiment 1 , despite the fact that in experiment 2 the participants received no feedback. People were able to use the information provided by mirror images to make judgments of the size and distance of the objects being reflected, even when no feedback and thus no learning was taking place. Again, as in experiment 1, the performance was far from perfect (see figure 3), but the participants did perform better than chance.

Experiment 2 also replicates the previous finding that distance judgments were more accurate than size judgments (ie steeper slope). Having shown that the result obtained in experiment 1 was not dependent upon feedback and thus learning, we decided to continue providing feedback in the remaining experiments.

\section{Experiment 3: Horizontal offset between object and its virtual image}

Experiments 1 and 2 showed that participants were able to make judgments of relative distance and size. It was important to assess exactly what information is important or critical to the judgments. Moreover, different factors may affect differently the size and the distance judgments. We hypothesised that better performance for distance judgments may be due to the presence of the additional cue of horizontal offset.

In the simulated scenes of experiments 1 and 2, a change in distance of the target sphere results in two changes in the image, a change in retinal size and also a correlated change in horizontal distance between the target sphere and its reflection. In other words, as the target objects gets closer to the mirror, the horizontal offset between it and its reflected image is reduced. In experiment 3 the apparent change in horizontal position associated with a change in depth was countered by the necessary change in horizontal position so that the edge of target sphere was always the same distance away from its reflected image. Figure 5 illustrates the change from experiments 1 and 2.

If the cue of horizontal offset is indeed responsible for the superior accuracy of the distance judgments over size judgments, then we should find that this difference is eliminated when the horizontal offset cue is eliminated. Note that the stimuli in experiment 1 are simulated photographs of an unfamiliar scene, but the stimuli in experiment 3 are doctored photographs designed to isolate a single source of information.

Although there was no effect of viewing condition in experiment 1 , it is conceivable that such an effect may be found with an increase in task difficulty. Therefore, we decided to include both monocular and binocular viewing conditions in experiment 3 .

\subsection{Method}

5.1.1 Participants, apparatus, and procedure. Sixteen Liverpool University undergraduates (who did not take part in the previous experiment) participated for course credits. The apparatus and procedure were identical to those in experiment 1, except for the nature of the test stimuli that used the same changes in depth and size but with the horizontal offset between target and reflection kept constant. The blue and yellow spheres were in slightly different locations relative to the mirror frame in comparison to experiment 1 (but they were always in the same location throughout the experiment). The yellow ball overlapped the frame by $4 \mathrm{~mm}$ for half the trials where its retinal size was $10 \%$ taller than that of the blue ball, and by $8 \mathrm{~mm}$ for the other half of the trials where its retinal size was $10 \%$ shorter. The blue ball overlapped the frame 

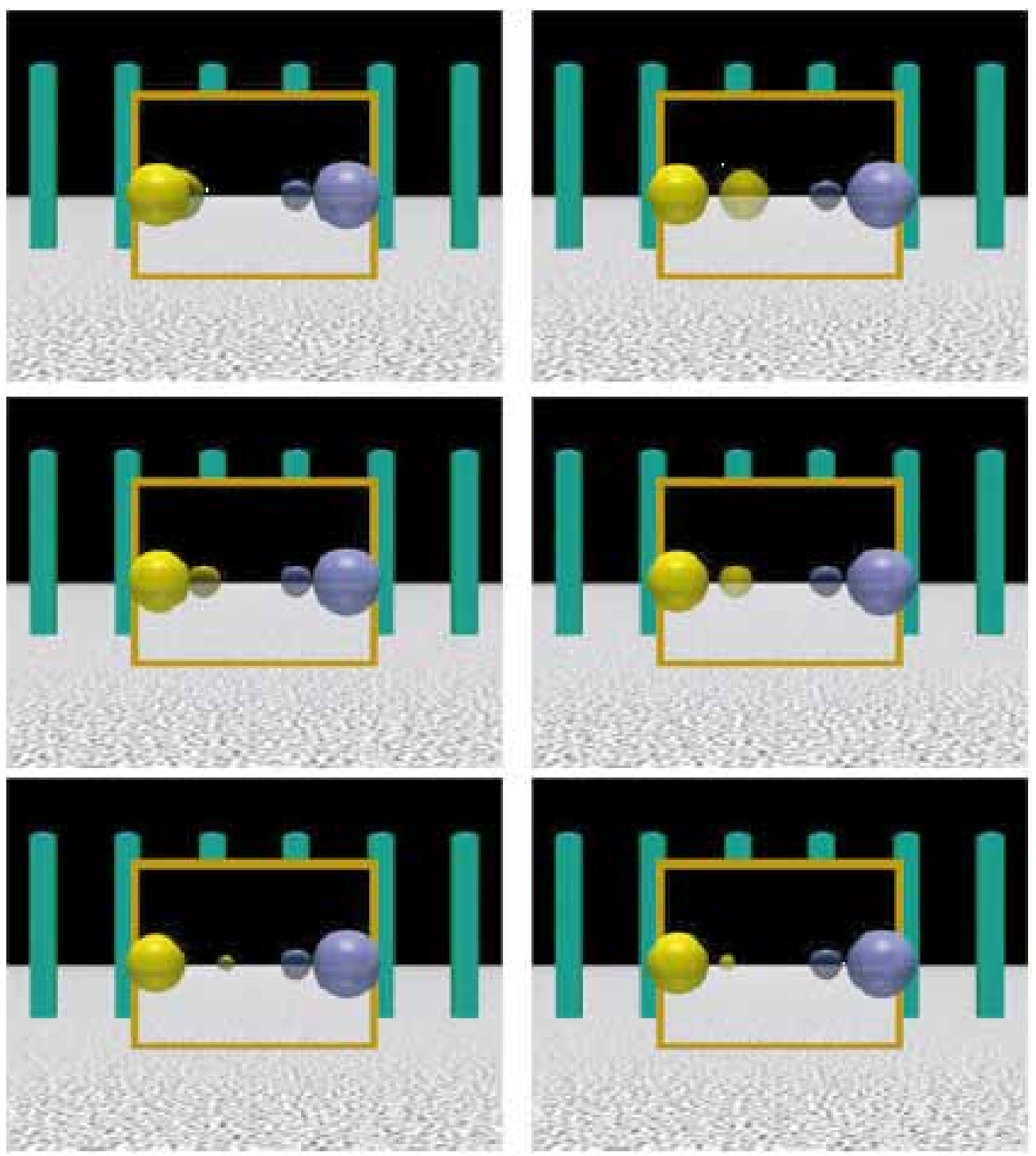

(a) Experiment 1

(b) Experiment 2

Figure 5. (a) Stimuli from experiment 1 with variable horizontal offset of the virtual and real sphere. (b) Stimuli from experiment 3, where horizontal offset is constant. The yellow sphere is at the same distance from viewpoint in the adjacent panels. The top panels show the yellow ball at its furthest distance from the viewpoint ( $40 \%$ farther than the blue ball), middle panels show the yellow ball $10 \%$ farther away than the blue ball, and the bottom panels show the yellow ball at its nearest point to the viewpoint ( $40 \%$ closer than the blue ball).

by $5 \mathrm{~mm}$ in all trials. This was to negative problems with doctoring the images to eliminate the horizontal offset caused by overlap of the target and its reflection. The overlap of the balls and the mirror frame was never informative of size or distance.

\subsection{Results}

5.2.1 Size judgments. Figure 6a shows mean perceived size difference plotted against actual size difference. This figure shows that perceived size difference increased orderly and linearly with actual size difference. There also appears to be little effect of viewing condition (monocular versus binocular) on size judgments. 

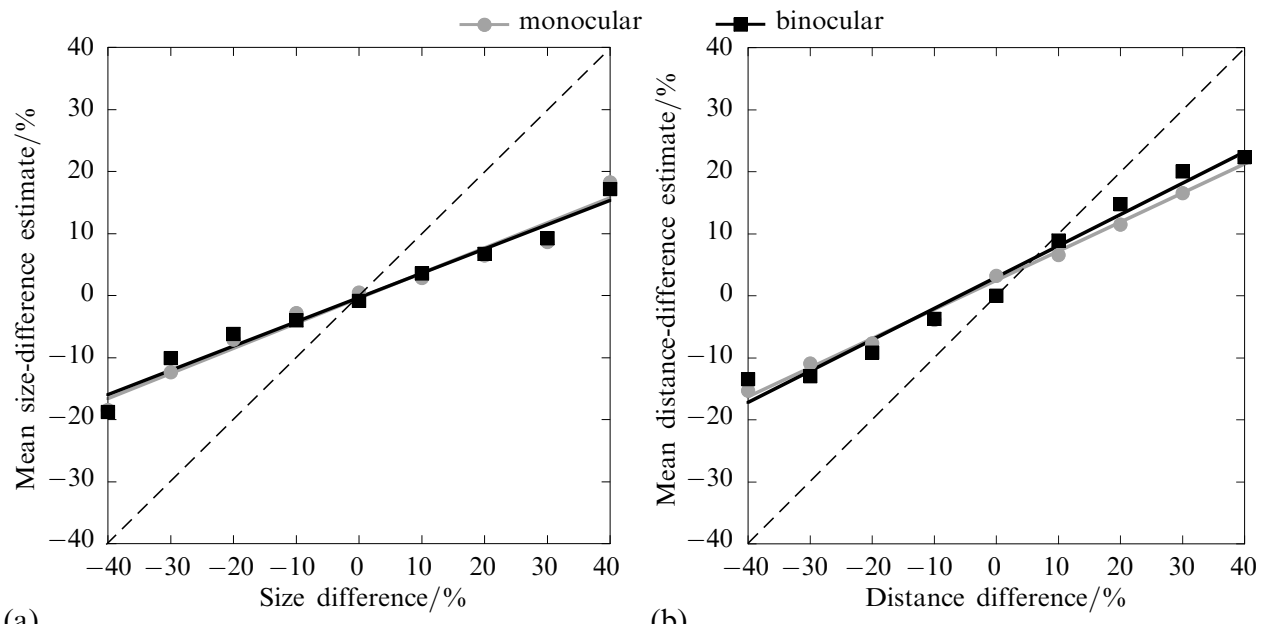

(b)

Figure 6. Plot of mean difference estimate against actual difference from experiment 3: (a) and (b) show size judgments and distance judgments, respectively. Data are fitted by linear regression. Slope values: monocular size 0.40 ; binocular size 0.39 ; monocular distance 0.46 ; binocular distance 0.50 .

These suggestions were confirmed by a repeated-measures ANOVA with viewing (monocular or binocular), and size difference $(-40 \%$ to $+40 \%$, in $10 \%$ steps) as factors. Size difference was analysed in the design as a linear contrast, as we were interested in the slope of the function. There was a significant effect of size difference $\left(F_{1,15}=61.36, p<0.001\right)$, indicating that participants were sensitive to this information. There was no significant effect of viewing $\left(F_{1,15}=0.01, p=0.86\right)$. There was also no significant viewing $\times$ size-difference interaction $\left(F_{1,15}=0.05, p=0.83\right)$, which indicated that the slopes for the monocular and binocular conditions did not differ significantly from each other.

5.2.2 Distance judgments. Figure $6 \mathrm{~b}$ shows mean perceived distance difference plotted against actual distance difference. This figure shows that mean perceived distance difference increased orderly and linearly with actual distance difference. There also appears to be little effect of viewing condition on distance judgments.

These suggestions were confirmed by a repeated-measures ANOVA with viewing (monocular or binocular) and distance difference ( $-40 \%$ to $+40 \%$, in $10 \%$ steps) as factors. As in the case of size analysis, the factor distance was treated as a linear contrast. There was a significant effect of distance difference $\left(F_{1,15}=50.20, p<0.001\right)$. There was no significant main effect of viewing condition $\left(F_{1,15}=1.03, p=0.033\right)$, and no significant viewing $\times$ distance-difference interaction $\left(F_{1,15}=0.81, p=0.380\right)$. This indicates that the slopes of the monocular and binocular conditions did not differ significantly from each other.

5.2.3 Distance versus size judgments. A comparison of the panels in figure 6 suggest that there is little difference between the slopes for the distance judgments and the size judgments. This suggestion was supported by a repeated-measures ANOVA with the task (size or distance), viewing (monocular or binocular), and distance/size-difference $(-40 \%$ to $+40 \%$, in $10 \%$ steps) as factors. As in the previous analyses, the factor distance $/$ size was treated as a linear contrast. There was a main effect of task $\left(F_{1,15}=11.94, p<0.01\right)$ but there was no significant task $\times$ distance/size-difference interaction $\left(F_{1,15}=1.46\right.$, $p=0.25$ ), indicating that the slopes for the distance and size judgments were not significantly different. There were no other significant main effects or interactions with 
the exception of a main effect of size/distance-difference $\left(F_{1,15}=84.78, p<0.01\right)$, which simply indicates that participants were sensitive to this information.

5.2.4 Experiment 1 versus experiment 3. The graphs in figures 3 and 6 seem to indicate that the slopes are steeper for experiment 1 than for experiment 3 . We therefore compared slopes for size and distance judgments across the two experiments, and as there was no effect of viewing condition in either experiment, we collapsed results across the task type.

5.2.5 Size judgments (experiment 1 versus experiment 3). A mixed ANOVA was carried out with experiment ( 1 or 3$)$ and the linear contrast of size difference $(-40 \%$ to $+40 \%$, in $10 \%$ steps $)$ as factors. There was a main effect of experiment $\left(F_{1,15}=10.72, p<0.01\right)$, but there was no significant experiment $\times$ size-difference interaction $\left(F_{1,15}=1.66\right.$, $p=0.22$ ), indicating that the slopes for the size judgments of experiments 1 and 3 were not significantly different from each other. There was again a significant effect of size/distance-difference $\left(F_{1,15}=84.78, p<0.01\right)$, which simply reflects the finding that participants were sensitive to this information.

5.2.6 Distance judgments (experiment 1 versus experiment 3). A mixed ANOVA was carried out with experiment (1 or 3), and the linear contrast of distance difference $(-40 \%$ to $+40 \%$, in $10 \%$ steps) as factors. There was a main effect of experiment $\left(F_{1,15}=11.10, p<0.01\right)$ and there was also a significant experiment $\times$ distancedifference interaction $\left(F_{1,15}=47.87, p<0.001\right)$ indicating that the slopes for the distance judgments of experiments 1 and 3 were significantly different from each other. There was again a significant effect of distance difference $\left(F_{1,15}=7.85, p<0.05\right)$, which simply reflects the finding that participants were sensitive to this information.

\subsection{Discussion}

The key finding from experiment 3 is that the difference in slopes between the distance and size judgments previously found in experiments 1 and 2 was eliminated with the elimination of horizontal offset. A comparison of experiments 1 and 3 shows that the removal of the horizontal offset did not alter the accuracy of size judgments but did reduce the accuracy of distance judgments, eliminating the previously found superior performance over size judgments. These results are consistent with our hypothesis that the extra cue of horizontal offset was responsible for the difference in size and distance judgment accuracy found in experiment 1 .

Another finding from experiment 3 is that there was no effect of viewing condition, even though the drop in distance-judgment performance shows that there was an increase in task difficulty. There are two explanations why we have failed to find an effect of viewing condition in experiments 1 and 3. First, it may be that the presence of the reduction screen is sufficient to increase the perceived depth in the binocular condition to a level comparable with monocular viewing. Second, even if apparent depth is significantly greater in the monocular condition, performance essentially depends on the relationship of the images of the objects and their reflections. This is a monocular cue that is retained in flat pictures.

\section{Experiment 4: Stereograms with and without mirror present; binocular disparity present in mirror reflection}

We have found that people can use information from reflections when other cues for depth and size had been removed. But, we would also like to know if the presence of reflections can contribute to size and distance judgments for scenes in which other depth cues are present.

In experiment 4 we used stimuli similar to those in experiment 1 , but for each display we produced a pair of stereograms. Examples are given in figure 7. The offset between 
the left and right views was $32.5 \mathrm{~mm}$ (half the average interocular distance). Observers were approximately placed at the correct viewpoint. Binocular disparity does provide metric information only if the distance from the viewpoint is correctly perceived (Howard and Rogers 2002). We cannot assume that this was the case, but what is important is that binocular information completely disambiguates the scene in terms of which sphere is nearer and which sphere is bigger. Moreover, the amount of disparity correlates with the amount of difference that observers are asked to judge, making the task relatively easy.

To test any advantage for the presence of reflections, we compared two conditions. The first condition contained a mirror in the scene as in experiment 1 . The second condition contained a blackboard located at the location of the mirror. Simply turning off the specularity of the mirror surface created the blackboard condition.

If reflections provide information even under stereoscopic viewing then we would expect size and distance judgments to be significantly more accurate in the mirror condition than in the blackboard condition.

\subsection{Method}

6.1.1 Participants. Eighteen Liverpool University undergraduates (who did not take part in the previous experiments) participated for course credits. They were screened for normal stereo vision with the TNO test for stereoscopic vision. The acuity ranged between 15 and $120 \mathrm{~s}$ of arc. Each participant wore a pair of stereoscopic glasses throughout the experiment.

6.1.2 Apparatus and procedure. The stimuli were similar to those used in experiment 1 . We generated stereograms by taking images of the scene from two slightly different vantage points, using the average human interocular distance $(65 \mathrm{~mm})$. These stereograms were presented on a Sony F500T9 monitor with a resolution of $1280 \times 1024$ pixels at $120 \mathrm{~Hz}$. Two stereo images were presented with the use of a NuVision infrared emitter and stereoscopic glasses. The effect of interleaving left and right images was that effective vertical resolution and refresh rate were halved $(512$ pixels at $60 \mathrm{~Hz})$. This describes the creation and presentation of the mirror stimuli. The blackboard stimuli were identical except that in the simulation the specularity of the mirror surface was turned off to create a matt black surface (figure 7).

Participants completed a distance-judgment condition and a size-judgment condition sequentially and in a counterbalanced order. Within each condition the blackboard and mirror trials were randomly interleaved.

\subsection{Results}

6.2.1 Mirror versus blackboard size judgments. Inspection of figure 8a appears to show a steeper gradient for the mirror condition than for the blackboard condition. This suggestion was confirmed by a repeated-measures ANOVA with size difference $(-40 \%$ to $+40 \%$, in $10 \%$ steps), and condition (mirror or blackboard stimuli) as factors. As for the previous analyses the factor design was treated as a linear contrast. There was a main effect of condition $\left(F_{1,17}=4.89, p<0.05\right)$, and also a significant sizedifference $\times$ condition interaction $\left(F_{1,17}=15.22, p<0.01\right)$, indicating that the slope gradients for the size judgments in the mirror and blackboard conditions were significantly different. There was also a significant effect of size difference which confirms previous analyses that people were sensitive to size information in the stimuli $\left(F_{1,17}=127.11, p<0.001\right)$.

6.2.2 Mirror versus blackboard distance judgments. Inspection of figure 8b appears to show a steeper slope for the mirror than for the blackboard conditions. This suggestion was confirmed by a repeated-measures ANOVA with distance difference $(-40 \%$ to $+40 \%$, in $10 \%$ steps), and condition (mirror or blackboard stimuli) as factors. As for the previous 


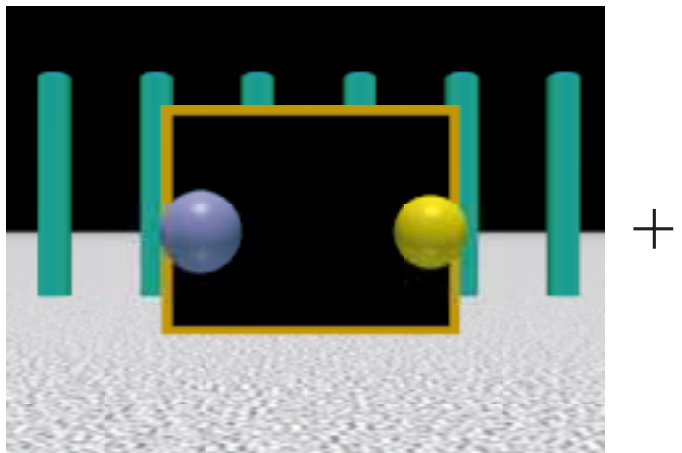

left eye image

(a)

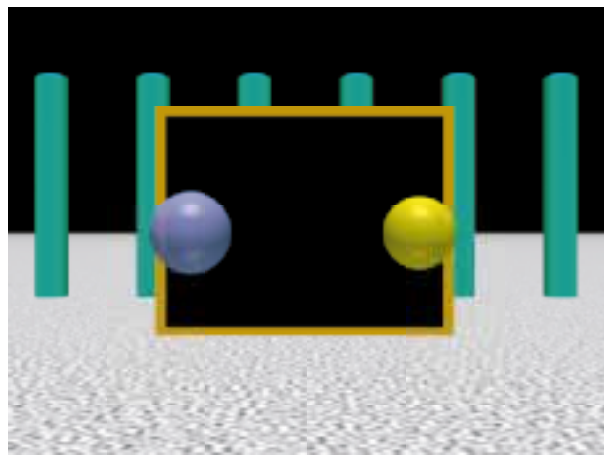

right eye image

Blackboard condition

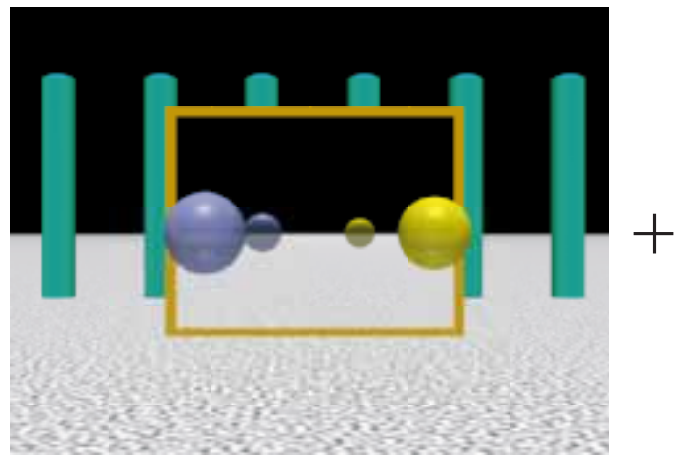

(b) left eye image

Mirror condition

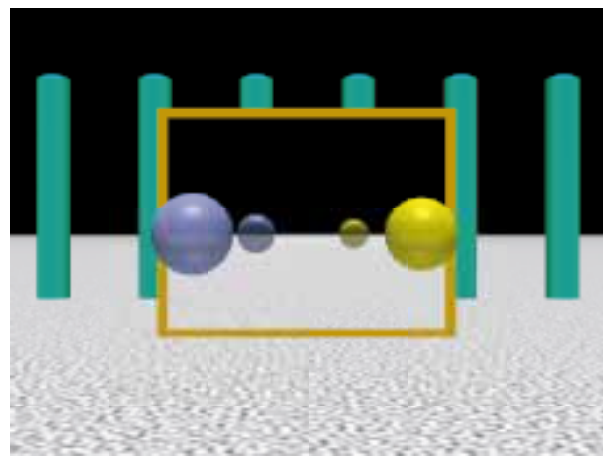

right eye image

Figure 7. Example of stereograms from experiment 4. The left-hand panels show the left-eye image, the right-hand panels show the right-eye image, for the blackboard condition (a) and mirror condition (b).

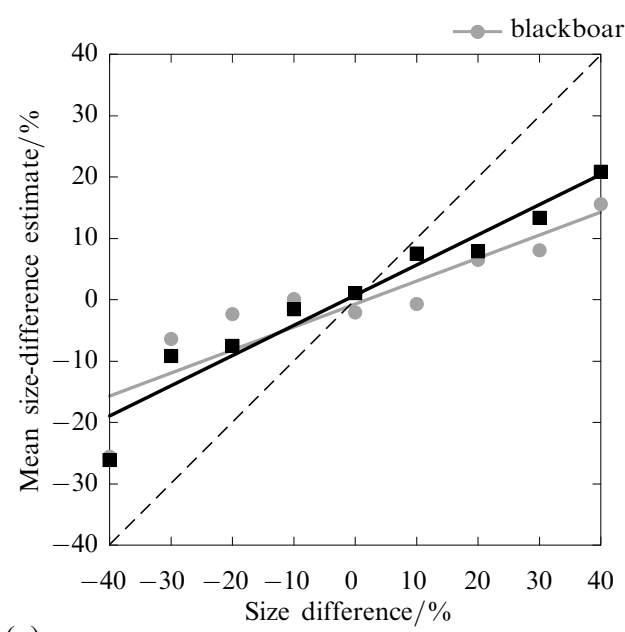

(a)

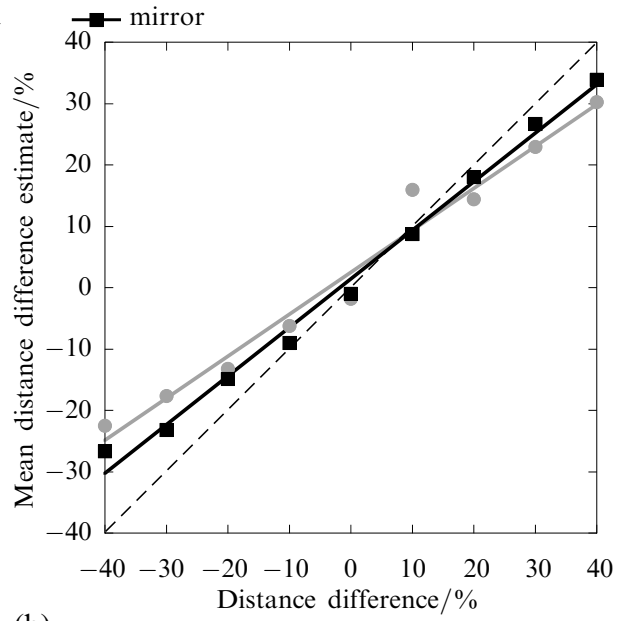

(b)

Figure 8. Plot of mean difference estimate against actual difference from experiment 4: (a) and (b) show size judgments and distance judgments, respectively. Data are fitted by linear regression. Slope values: blackboard size 0.37 ; mirror size 0.49 ; blackboard distance 0.68 ; mirror distance 0.79 . 
analyses the factor design was treated as a linear contrast. There was no main effect of condition $\left(F_{1,17}=2.44, p=0.137\right)$; however, there was a significant distancedifference $\times$ condition interaction $\left(F_{1,17}=14.11, p<0.01\right)$, indicating that the slope gradient for the size judgments in the mirror and blackboard conditions were significantly different. There was also a significant effect of distance difference which confirms previous analyses that people were sensitive to size information in the stimuli $\left(F_{1,17}=488.52, p<0.001\right)$.

6.2.3 Size versus distance judgments. Inspection of figure 8 appears to show a steeper slope for distance judgments than for size judgments for both the mirror and blackboard conditions. This was confirmed by a repeated-measures ANOVA with task (size or distance) and distance/size-difference ( $-40 \%$ to $+40 \%$, in $10 \%$ steps) as factors. As for the previous analyses, the factor design was treated as a linear contrast. There was a significant main effect of task for the blackboard condition $\left(F_{1,17}=13.58\right.$, $p<0.01)$, but not for the mirror condition $\left(F_{1,17}=4.28, p=0.522\right)$. However, the key finding is that there was a significant task $\times$ distance/size-difference interaction for both the blackboard condition $\left(F_{1,17}=38.58, p<0.001\right)$, and the mirror condition $\left(F_{1,17}=39.36, p<0.001\right)$. There was also a significant effect of size/distance-difference for both the blackboard condition $\left(F_{1,17}=571.50, p<0.001\right.$, and the mirror condition $\left(F_{1,17}=306.87, p<0.001\right)$, which confirms previous analyses that people were sensitive to size and distance information in the stimuli.

6.2.4 Experiment 4 versus experiment 1. To assess the impact of the presence of binocular disparity information in the image we compared the results of experiments 1 and 4 . As there was no effect of viewing condition (monocular and binocular) in experiment 1 , we collapsed the data across viewing conditions.

6.2.5 Mirror size judgments (experiment 4 versus experiment 1). A repeated-measures ANOVA was carried out with size difference ( $-40 \%$ to $+40 \%$, in $10 \%$ steps) as a withinsubjects factor and experiment (1 or 4) as a between-subjects factor. As for previous analyses, the factor design was treated as a linear contrast. There was no main effect of experiment $\left(F_{1,32}=1.29, p=0.27\right)$, nor was there a significant experiment $\times$ size-difference interaction $\left(F_{1,32}=1.71, p=0.20\right)$, indicating that the slope for the size condition of experiment 4 was not significantly different from that of experiment 1 . Again there was a significant effect of size difference $\left(F_{1,32}=111.73, p<0.001\right)$, supporting all previous analyses that people were sensitive to this information.

6.2.6 Mirror distance judgments (experiment 4 versus experiment 1). A repeated-measures ANOVA was carried out with distance difference ( $-40 \%$ to $+40 \%$, in $10 \%$ steps) as a within-subjects factor and experiment ( 1 or 4$)$ as a between-subjects factor. There was no main effect of experiment $\left(F_{1,32}=2.23, p=0.15\right)$, but there was a significant experiment $\times$ distance-difference interaction $\left(F_{1,32}=184.99, p<0.001\right)$. Again there was a significant effect of distance difference $\left(F_{1,32}=52.44, p<0.001\right)$, supporting all previous analyses that people were sensitive to this information.

\subsection{Discussion}

The results of experiment 4 show greater accuracy of depth and size judgments when mirror reflections are visible, even in the presence of binocular disparity. This finding complements the findings of experiment 1 and 4 . In experiments 1 and 3 we found that people could use reflection information when it was the only information available; experiment 4 demonstrated that reflections are spontaneously used by observers, even when the task could be completed by ignoring the reflection information. 
Additionally, the comparison of the results from experiments 1 and 4 shows that the presence of binocular disparity in experiment 4 increased the steepness of the slopes for distance judgments but not for size judgments.

It is unlikely that the observer perceived the exact absolute position in space of the blue and yellow balls, as this would depend on accurate distance perception and perhaps also require a stricter enforcement of correct viewing position (around $25 \mathrm{~cm}$ ) through the use of a chin-rest. Nonetheless, the binocular disparity information is still suitably rich to provide information for the relative judgments that we required. Moreover, the real focus of our study was a comparison between the condition in which the mirror was present and that in which the blackboard was present, as binocular information was identical in both.

\section{Experiment 5: Stereograms with and without mirror present; binocular disparity absent in mirror reflection}

Experiment 4 showed that, even with the presence of binocular disparity for a visual scene, the information afforded by reflections improves participants' size and distance judgments. This is a surprising result: whereas experiments $1-3$ demonstrated that people use mirror reflections for depth and size judgments in the absence of other information, experiment 4 shows that people use the additional information afforded by a mirror even when information from binocular disparity is present.

There is a complication in fully interpreting the findings of experiment 4 . When we introduced binocular disparity to the visual scene in experiment 4 we obviously introduced disparity for the virtual objects as well as for the target objects. Thus, not only were monocular cues in the mirror present as in experiments $1-3$, but also disparity cues from the virtual objects; this disparity was correlated with the distance of the target objects from the viewpoint, and could have been used by the participants. Experiment 5 was devised to investigate the role of disparity in the mirror reflection.

We created a new set of stereograms for experiment 5 , identical to the stereograms used in experiment 4 except that the disparity in the mirror was removed. Again there were a blackboard and a mirror conditions. These stimuli have the monocular information based on the ratio of the height of the target and its reflection, but the disparity information of the virtual targets was removed. These stimuli show two spheres in front in a flat picture, but the picture is special in that it contains the monocular information that would be present in a mirror. If people use the monocular information from the mirror, then they will perform significantly better in the mirror condition than in the blackboard condition for both size and distance judgments.

\subsection{Method}

7.1.1 Participants. Eighteen Liverpool University undergraduates (who did not take part in the previous experiments) participated for course credits. They were screened for normal stereo vision using the TNO test for stereoscopic vision. The acuity ranged between 15 and $240 \mathrm{~s}$ of arc. Each participant wore a pair of stereoscopic glasses throughout the experiment.

7.1.2 Apparatus and procedure. The stimuli were similar to those used in experiment 4. The only difference was in the mirror condition stimuli where the binocular disparity present for the virtual objects and scene in the mirror was removed; disparity for the remainder of the scene was left in place. As for experiment 4, the experiment was separated into a distance judgment condition and a size judgment condition. Participants completed both tasks in a counterbalanced order. Within each condition the blackboard and mirror trials were randomly interleaved. 


\subsection{Results}

7.2.1 Mirror versus blackboard size judgments. Inspection of figure 9 appears to show a steeper gradient for the mirror condition than for the blackboard condition. This suggestion was confirmed by a repeated-measures ANOVA with size difference ( $-40 \%$ to $+40 \%$, in $10 \%$ steps), and condition (mirror or blackboard stimuli) as factors. As for the previous analyses, the factor design was treated as a linear contrast. There was a main effect of condition $\left(F_{1,17}=8.82, p<0.01\right)$, and also a significant size-difference $\times$ condition interaction $\left(F_{1,17}=6.48, p<0.05\right)$, indicating that the slope gradient for the size judgments in the mirror and blackboard conditions are significantly different. There was also a significant effect of size difference which confirms previous analyses that people were sensitive to size information in the stimuli $\left(F_{1,17}=102.01, p<0.001\right)$.
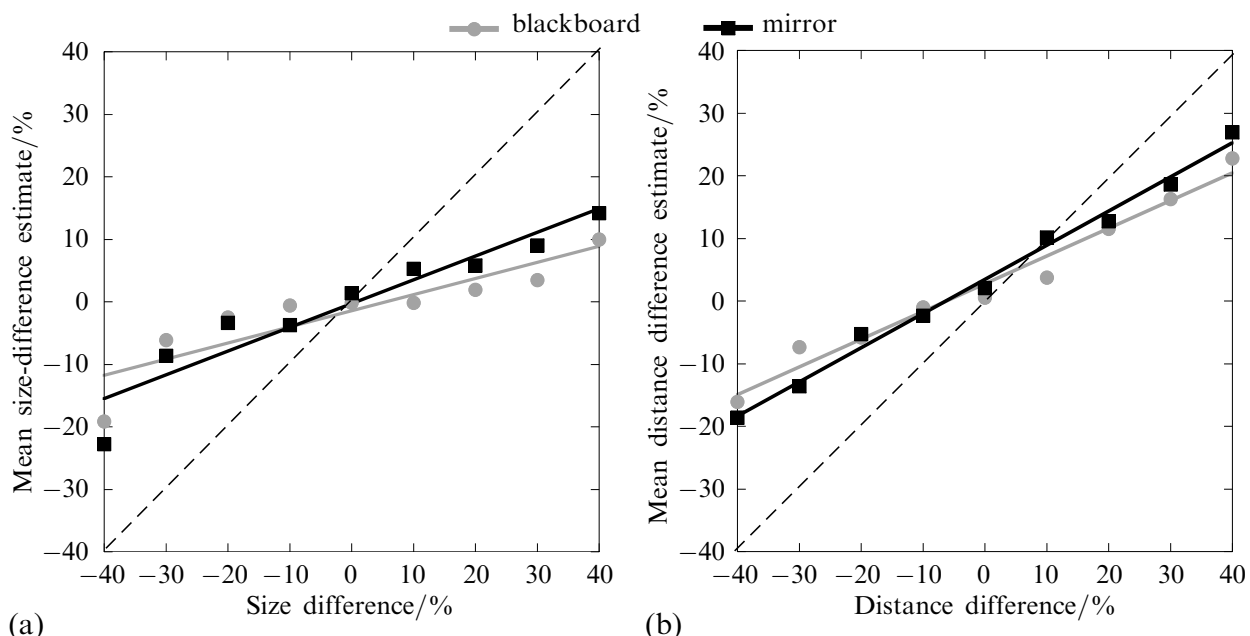

Figure 9. Plot of mean difference estimate against actual difference from experiment 5: (a) and (b) show size judgments and distance judgments, respectively. Data are fitted by linear regression. Slope values: blackboard size 0.26 ; mirror size 0.38 ; blackboard distance 0.44; mirror distance 0.55 .

7.2.2 Mirror versus blackboard distance judgments. Inspection of figure 9 appears to show a steeper slope for the mirror condition than for the blackboard condition. This suggestion was confirmed by a repeated-measures ANOVA with distance difference ( $-40 \%$ to $+40 \%$, in $10 \%$ steps), and condition (mirror or blackboard stimuli) as factors. As for the previous analyses, the factor design was treated as a linear contrast. There was no main effect of condition $\left(F_{1,17}=0.29, p=0.59\right)$; however, there was a significant distance-difference $\times$ condition interaction $\left(F_{1,17}=6.07, p<0.05\right)$, indicating that the slope gradient for the size judgments in the mirror and blackboard conditions are significantly different. There was also a significant effect of distance difference which confirms previous analyses that people were sensitive to distance information in the stimuli $\left(F_{1,17}=104.34, p<0.001\right)$.

7.2.3 Size versus distance judgments. Inspection of the left and right panels of figure 9 appears to show a steeper slope for distance judgments than for size judgments for both the mirror and blackboard conditions.

This was confirmed by a repeated-measures ANOVA with task (size or distance) and distance/size-difference ( $-40 \%$ to $+40 \%$, in $10 \%$ steps) as factors. As for the previous analyses the factor design was treated as a linear contrast. There was a significant main effect of task for both the blackboard condition $\left(F_{1,17}=13.15, p<0.01\right)$, and the 
mirror condition $\left(F_{1,17}=8.84, p<0.01\right)$. There was also a significant task $\times$ distance/ size-difference interaction for both the blackboard condition $\left(F_{1,17}=17.92, p<0.001\right)$, and the mirror condition $\left(F_{1,17}=4.50, p<0.05\right)$. There was also a significant effect of size/distance-difference for both the blackboard condition $\left(F_{1,17}=146.79, p<0.001\right)$, and the mirror condition $\left(F_{1,17}=94.60, p<0.001\right)$, which confirms that people were sensitive to size and distance information.

\subsection{Discussion}

Experiment 5 shows that the monocular information from the mirror is used by observers even when the binocular disparity information is removed from the virtual objects but is still present for the target objects. As for experiment 4, the key result is the significant difference in slope between the mirror and blackboard conditions, with a steeper slope for the mirror condition for both the size and distance tasks. Experiments $1-3$ demonstrated that people use mirror reflections to make size and distance judgments in the absence of other salient cues. Experiments 4 and 5 show that people still make use of the mirror information even when the informative cue of binocular disparity is present for the target objects.

An additional finding from experiment 5 was that the slopes for distance judgments in both the mirror and blackboard conditions were steeper than those for the size judgments. This again supports the results of the previous experiments.

\section{General discussion}

Overall, the results of our experiments tell a consistent story. Experiment 1 shows that, in the absence of the usual cues to size and distance, people can use the relationship between an object and its reflection to disambiguate a visual scene and make above-chance judgments of size and distance. A significant advantage for distance judgments over size judgments was found in experiment 1 . We suggested that this was due to the additional cue of horizontal offset between the real and virtual object, which is salient for distance but not size. This suggestion was supported by the results of experiment 3 in which the advantage for distance judgments was eliminated. When we compared directly the results of experiments 1 and 3, the judgments of size did not differ significantly between the two experiments, while the judgments of distance were more accurate in experiment 1 where horizontal offset was present.

Experiment 4 shows that even under richer viewing conditions, when binocular disparity was present, there was a significant advantage for scenes in which the additional information from reflections was available. This was clear from the difference in slopes between the mirror and blackboard conditions. Additionally, the accuracy of distance judgments was higher in experiment 4 (with binocular disparity) than in experiment 1 (without disparity), whilst size judgments did not differ.

In experiment 5, when binocular disparity information was absent for the virtualobjects, performance was still more accurate in the mirror condition than in the blackboard condition. This supports the idea that the monocular information provided by the reflections (relationship between the object and its reflection) is the critical information.

In experiment 2 we controlled for the presence of directional feedback and found that participants could still perform both the size and distance judgments at an above-chance level. This finding rules out the possible objection that learning within the experiment was the sole cause of the above-chance performance. The finding of a steeper slope for distance judgments compared to size judgments in the presence of horizontal offset was also replicated.

If we accept that observers use the reflection information, then a number of new questions arise. There is an apparent conflict between the use of reflections in our studies, 
and the systematic errors that are found when people are asked about reflections more generally. In the naive optics literature it is a common finding that people are inaccurate about how much of the environment is visible in a mirror (Bertamini et al 2003b), and people are generally insensitive to the viewpoint when considering what is visible in a mirror (Bertamini et al 2003a; Bertamini and Parks 2005). Reasoning about what is made visible in a mirror, and using what is visible in a mirror to make judgments about visual space appear to be divorced domains. To understand the literature, one has to remember that different results stem from key differences in experimental situations. In one type of situation people are making predictions about mirror reflections rather than judgments based on their contents; here people are not accurate because they have little knowledge of what is visible from different viewpoints (eg Bertamini et al 2003b). In another situation, which was studied more recently, people attempt to judge properties of the projections on the mirror surface. Here people are not accurate even when the mirror is in front of them because they cannot see a transparent surface as opaque (Bertamini et al 2007; Lawson et al 2007). The third type of situation is when people are making judgments about scenes (ie distal objects, not projections). This is the task studied in the series of experiments reported here. We found that people perform with considerable accuracy. There is an interesting parallel with what has been found with shadows. People do not notice shadows as such, just like people do not notice reflections. Yet shadows, and reflections, can also affect which objects are perceived, their shape and location (eg Mamassian et al 1998).

Although the prevalence and experience of planar mirrors has occurred late in human evolution, our participants showed that they had acquired the ability to use some information from mirror reflections. This suggests that perhaps this ability is learnt. Future research could examine this issue from a developmental perspective. If this skill is learned one should predict differential performance between people who have more or less experience of mirror reflections.

Finally, our studies only show that under some conditions reflections affect human perception of size and distance; we do not know whether reflections remain powerful under more general conditions.

\section{Conclusions}

We have explored whether the presence of a mirror reflection in a scene can enrich the perception of distance and size of unfamiliar objects. This approach is different from previous work which has focused on people's understanding of mirror reflections (eg Bertamini et al 2003b; Bertamini and Parks 2005). People can use information from reflections to make judgments about the relative position and size of the objects reflected. Although we found that mirror reflections are a source of information about depth and size for human observers, we need to qualify this result. Our stimuli are special in that two objects are present side by side, and a planar mirror is present in the frontoparallel plane. Even under these conditions performance was above chance but far from perfect. Future work will need to pit this type of information against other cues to establish its relative strength.

\section{References}

Ames A Jr, 1925 "The illusion of depth from single pictures" Journal of the Optical Society of America $10137-148$

Bertamini M, Latto R, Spooner A, 2003a "The Venus effect: people's understanding of mirror reflections in paintings" Perception $32593-599$

Bertamini M, Lawson R, Liu D, 2007 "Understanding 2D projections on mirrors and on windows" Spatial Vision in press

Bertamini M, Parks T E, 2005 "On what people know about images on mirrors" Cognition 98 $85-104$ 
Bertamini M, Spooner A, Hecht H, 2003b "Naive optics: Predicting and perceiving reflections in mirrors" Journal of Experimental Psychology: Human Perception and Performance $29982-1002$

Bertamini M, Yang T, Proffitt D R, 1998 "Relative size perception at a distance is best at eye level" Perception \& Psychophysics $60673-682$

Croucher C J, Bertamini M, Hecht H, 2002 "Naive optics: Understanding the geometry of mirror reflections" Journal of Experimental Psychology: Human Perception and Performance $28546-562$

Elder J H, Trithart S, Pintilie G, MacLean D, 2004 "Rapid processing of cast and attached shadows" Perception $331319-1338$

Epstein W, Park J, Casey A, 1961 "The current status of the size-distance hypotheses" Psychological Bulletin $58491-514$

Fleming R, Dror R, Alderson E H, 2003 "Real-world illumination and the perception of surface reflectance properties" Journal of Vision 3347 - 368

Gallup G G Jr, 1998 "Can animals empathise?” Scientific American Presents 9 66-71

Gardner M, 1967 The Ambidextrous Universe (New York: Basic Books)

Gregory R, 1997 Mirrors in Mind (London: Penguin)

Gruber H E, 1954 "The relation of perceived size to perceived distance" American Journal of Psychology $67411-426$

Hecht H, Bertamini M, Gamer M, 2005 "Naive optics: Acting upon mirror reflections" Journal of Experimental Psychology: Human Perception and Performance 31 1023-1038

Higashiyama A, Shimono K, 2004 "Mirror vision: Perceived size and perceived distance of virtual images" Perception \& Psychophysics 66 679-691

Howard I P, Rogers B J, 2002 Seeing in Depth (Toronto, Canada: Porteous)

Ittelson W H, 1993 "Mirror reversals: real and perceived" Perception 22 855-861

Ittelson W H, Mowafy L, Magid D, 1991 "The perception of mirror-reflected objects" Perception $20567-584$

Jones L A, Bertamini M, 2007 "The eyes have it: The naive beliefs about reflections" Quarterly Journal of Experimental Psychology under revision

Kleffner D A, Ramachandran V S, 1992 "On the perception of shape from shading" Perception \& Psychophysics $\mathbf{5 2} 18-36$

Knill D C, Mamassian P, Kersten D, 1997 "The geometry of shadows" Journal of the Optical Society of America A 14 3216-3233

Lawson R, Bertamini M, Liu D, 2007 "Images on mirrors and windows cannot be perceived" Journal of Experimental Psychology: Human Perception and Performance 351027 - 1044

Nicholls A L, Kennedy J M, 1993 "Foreshortening and the perception of parallel projections" Perception \& Psychophysics $54665-674$

Mamassian P, Knill D C, Kersten D, 1998 "The perception of cast shadows" Trends in Cognitive Sciences $2288-295$

Povinelli D J, 2000 Folk Physics for Apes (Oxford: Oxford University Press)

Rogers S, 2003 "Truth and meaning in pictorial space", in Looking into Pictures: An Interdisciplinary Approach to Pictorial Space Eds H Hecht, R Schwartz, M Atherton (Cambridge, MA: MIT Press) pp $301-320$

Sedgwick A, 1983 'Environment-centered representation of spatial layout', in Human and Machine Vision Eds J Beck, B Hope, A Rosenfeld (New York: Academic Press) pp 425-458

Schlosberg H, 1941 "Stereoscopic depth from single pictures" American Journal of Psychology 54 $601-605$

Warton E, 1902 "Vesalius in Zante (1564)" North American Review 175 625-631

Yang T, Kubovy M, 1999 "Weakening the robustness of perspective: Evidence for a modified theory of compensation in picture perception" Perception \& Psychophysics $61456-467$ 


\section{PERTEPTION}

VOLUME 362007

www.perceptionweb.com

Conditions of use. This article may be downloaded from the Perception website for personal research by members of subscribing organisations. Authors are entitled to distribute their own article (in printed form or by e-mail) to up to 50 people. This PDF may not be placed on any website (or other online distribution system) without permission of the publisher. 